AEI-2011-022

\title{
Near horizon data and physical charges of extremal AdS black holes
}

\author{
Dumitru Astefanesei ${ }^{a}$, Nabamita Banerjee ${ }^{b}$, Suvankar Dutta ${ }^{c}$ \\ ${ }^{a}$ Max-Planck-Institut für Gravitationsphysik, Albert-Einstein-Institut, 14476 Golm, Germany \\ ${ }^{b}$ ITF, Utrecht University, Utrecht, The Netherlands \\ ${ }^{c}$ Department of Physics, Swansea University, Swansea, U.K.
}

Email: dumitru@aei.mpg.de, N.Banerjee@uu.nl, pysd@swan.ac.uk

\begin{abstract}
We compute the physical charges and discuss the properties of a large class of fivedimensional extremal AdS black holes by using the near horizon data. Our examples include baryonic and electromagnetic black branes, as well as supersymmetric spinning black holes. In the presence of the gauge Chern-Simons term, the five-dimensional physical charges are the Page charges. We carry out the near horizon analysis and compute the four-dimensional charges of the corresponding black holes by using the entropy function formalism and show that they match the Page charges.
\end{abstract}

Keywords: AdS black holes, Entropy function, Page charges 


\section{Contents}

$\begin{array}{lll}1 & \text { Introduction } & 1\end{array}$

2 Near horizon geometry and physical charges $\quad 2$

3 Examples 6

3.1 Baryonic black branes . . . . . . . . . . . . . . . . . . . . . 6

3.2 Stationary black holes . . . . . . . . . . . . . . . . 8

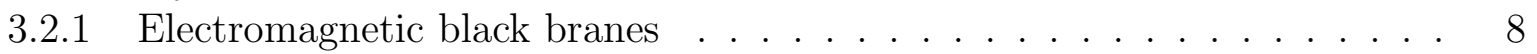

3.2.2 Susy spinning AdS black holes . . . . . . . . . . . . . . . . 10

4 Discussion 11

$\begin{array}{ll}\text { A KK reduction of Chern-Simons term } & 13\end{array}$

B Toroidal Spinning Black Brane $\quad 14$

\section{Introduction}

Black hole physics is a very active area of ongoing research. A statistical understanding of black hole entropy is one of the most important and long standing questions in theoretical physics.

In the last years, important progress in understanding the attractor mechanism and entropy of extremal (non-BPS) black holes was based on the entropy function formalism [1, 2, 3, 4]. An important advantage of this method is that one needs just the near horizon geometry to characterize the black hole. In particular, one can compute the entropy and the conserved charges by using just the near horizon geometry [4, 5].

In [5] it was explicitly shown how to construct the conserved charges of black holes/rings by using just the near horizon data. For example, it is by now well known that the dipole charge appears in the first law of black ring thermodynamics, even if it is not conserved [6]. Therefore, the entropy of the black rings can also depend on the dipole charges, not only on the usual conserved charges.

In general, when there are degrees of freedom living outside the horizon, they contribute to the asymptotic charges and so the asymptotic charges and the charges computed from the near horizon data may not be the same 1 One expects that the macroscopic entropy is completely determined by the near horizon geometry. However, a counterexample is the $4 D-5 D$ lift of BMPV black hole [8]. That is, the $4 D$ and $5 D$ black holes have the same near horizon geometry, but different microscopic spectra. If true, that would imply that different microscopic entropies will correspond to the same near horizon geometry. In fact, this discrepancy [9, 10] was solved in [11 by removing the 'hair', which contributes to the degeneracies. This further suggests that the physical charges can be obtained, in fact, from the near horizon data only.

\footnotetext{
${ }^{1} \mathrm{~A}$ discussion on asymptotic charges and the charges computed at the horizon for the black ring can be found in 7 .
} 
Also, in the presence of the gauge Chern-Simons term, there are subtleties in the definition of the charges. Since the Maxwell charge is carried by the gauge fields themselves, it is diffused throughout the bulk and so it is not localized. This clearly resembles the previous discussion, and one expects a 'hair' contribution to the asymptotic charges. The physical charges in this case are the so called 'Page charges' (see [12] for a nice brief review).

A similar analysis can be used for asymptotically AdS black holes. However, the interpretation of the attractor mechanism is quite different than in flat space. That is, the moduli flow is in fact an RG flow towards the IR attractor horizon once the theory is embedded in string theory (more precisely in type IIB) [13. Interestingly enough, the near horizon data can be also used to compute the shear viscosity coefficient, not just the entropy [14, 15, 16, 17, 2]

Given these motivations, it is clearly useful to understand the robustness of the near horizon analysis for the AdS black holes. In this paper we generalize the work of [5] to AdS black holes. Though, an important difference is that we use the entropy function formalism to obtain the four dimensional physical charges and compare them with the charges of [5]. We would like to emphasize that our analysis is built on the previous (but less general) work on asymptotically flat and AdS extremal black holes [5, 19, 20, 21]. However, the near horizon geometry ansatz we consider is more general due to the existence of a magnetic Kaluza-Klein (KK) part. We work out in detail several examples, which are relevant in the context of AdS/CFT duality.

The paper is organized as follows: in Section 2 we present a concrete analysis of the entropy function formalism for a generic near horizon geometry ansatz when the gauge Chern-Simons term is present. We also show that the four-dimensional charges obtained from the entropy function after KK reduction match the five-dimensional Page charges. In Section 3 we apply the general results of Section 2 for some concrete examples. Finally, we conclude with a discussion of our results. In Appendix A we present details of the KK reduction of the Chern-Simons term. Appendix B contains an analysis of toroidal spinning black branes.

\section{Near horizon geometry and physical charges}

In this section, we present a detailed analysis of the entropy function formalism for a general class of 5-dimensional AdS black holes. We start with an action that contains a Chern-Simons gauge term and, to compute the physical charges, we KK reduce to obtain a gauge invariant action in four dimensions. A similar analysis for asymptotically AdS and flat black holes was carried out in [19, 20] - for other applications of entropy function in AdS space, see [22]. However, our analysis is more general since we work with a generic KK field. In this way, we can study within the same set-up the SUSY AdS spinning black holes and, also, black branes and non-supersymmetric extremal black holes. The latter are important since yield insights into the physics of strongly interacting systems at non-zero density [23, 24, 17].

We start with a brief review of [5] and generalize these results to AdS black holes (to our best knowledge, an analysis for AdS black holes did not appear before). Then, we compare these results with the ones obtained from the entropy function formalism.

\footnotetext{
${ }^{2}$ For other transport coefficients there is, in general, a non-trivial flow and the near horizon data is not enough [18].
} 
Why an analysis of physical properties from the near horizon geometry is important? Since, in general, it is not easy to construct the full analytic black hole solutions in complicated theories, it is clearly very advantageous to extract information from the near horizon data only. For example, one can compute the viscosity/entropy ratio just with the knowledge of the near horizon geometry [16].

Another important application is in the case of black holes for which there exist degrees of freedom living outside the horizon ('hair') — from the point of view of an asymptotic observer, the hair can contribute to the macroscopic degeneracy and/or the asymptotic charges. However, the near horizon geometry should encode the 'statistical' information for computing the entropy (to compare the macroscopic degeneracy with the microscopic degeneracy, one has to subtract the hair, [11]). Therefore, a computation of the physical charges at the horizon is important.

Due to the presence of the gauge Chern-Simons term in the 5-dimensional theory, the usual 'Maxwell charge' is not localized - this charge is carried by the gauge fields themselves and so is diffused throughout the bulk. An important question then is what is the physical charge in the presence of Chern-Simons term? In [5] it was argued that, in fact, the so called 'Page charge' is the correct 5D charge. The Maxwell charges in 4D are obtained by KK reduction of the Page charges. We will use the entropy function formalism to show that, indeed, the 4D charges of [5] match the physical charges obtained from the entropy function formalism.

The theory we shall be considering is minimal $D=5$ gauged supergravity with bosonic action

$$
\begin{aligned}
S_{5} & =\frac{1}{4 \pi G_{5}} \int\left[\left(\frac{R_{5}}{4}+\frac{3}{\ell^{2}}\right) \star 1-\frac{1}{2} F \wedge \star F-\frac{2}{3 \sqrt{3}} F \wedge F \wedge A\right] \\
& =\frac{1}{\left(k_{5}\right)^{2}} \int d^{5} x\left[\sqrt{-g}\left(R_{5}-F^{2}+\frac{12}{\ell^{2}}\right)-\frac{2}{3 \sqrt{3}} \varepsilon^{\alpha \beta \gamma \tau \delta} A_{\alpha} F_{\beta \gamma} F_{\tau \delta}\right],
\end{aligned}
$$

where $R_{5}$ is the Ricci scalar, $F^{2} \equiv F_{\alpha \beta} F^{\alpha \beta}$, and $F=d A$ is the field strength of the $U(1)$ gauge field. We also use the notation $\left(k_{D}\right)^{2}=16 \pi G_{D}$, where $G_{D}$ is the gravitational constant in $D$ dimensions. The bosonic equations of motion are

$$
\begin{aligned}
{ }^{5} R_{\alpha \beta}-2 F_{\alpha \gamma} F_{\beta} \gamma+\frac{1}{3} g_{\alpha \beta}\left(F^{2}+\frac{12}{\ell^{2}}\right) & =0, \\
d * F+\frac{2}{\sqrt{3}} F \wedge F & =0 .
\end{aligned}
$$

The last equation can be rewritten as

$$
d\left(* F+\frac{2}{\sqrt{3}} A \wedge F\right)=* j=0
$$

and so it is this exterior derivative that should be identified with a current. It is clear that the 'Page' current is conserved and localized (in the usual sense that it vanishes when the equations of motion hold) — see [12] for a more detailed discussion.

Consequently, we can define a conserved, localized charge as the integral of the Page current over an arbitrary three-cycle, $\Sigma$ surrounding the black hole:

$$
\left(4 \pi G_{5}\right) Q_{5 d}=\int_{\Sigma}\left(* F+\frac{2}{\sqrt{3}} A \wedge F\right)=\int_{\infty}\left(* F+\frac{2}{\sqrt{3}} A \wedge F\right)=\int_{H}\left(* F+\frac{2}{\sqrt{3}} A \wedge F\right) .
$$


What is important for us is the fact that this charge can be computed using just the near horizon data [5], as can be easily seen from the above expression.

For spinning black holes a similar definition for the angular momentum exists — the expression of the angular momentum does not change in AdS:

$$
J_{\xi}=\frac{1}{16 \pi G_{5}} \int_{H}\left[* \nabla \xi+4 *(\xi \cdot A) F+\frac{16}{3 \sqrt{3}}(\xi \cdot A) A \wedge F\right] .
$$

Here, we keep the notations of [5]: $\xi$ is the axial Killing vector and $\nabla \xi$ is an abbreviation for the two-form $\nabla_{\mu} \xi_{\nu} d x^{\mu} \wedge d x^{\nu}=d \xi$.

One problem with these five-dimensional definitions is that the Page charges are not gauge invariant (under large gauge transformations). We note that, in fact, the Page charge differs from the usual Maxwell charge just by a boundary term. If this boundary term vanishes (e.g., when the gauge potential decays sufficiently fast at the boundary) the gauge freedom is removed and so a discussion in terms of Maxwell charges (computed at the boundary) is equivalent: $\int_{\infty} * F=\int_{\infty}\left(* F+\frac{2}{\sqrt{3}} A \wedge F\right)$. The supersymmetric spinning black holes of Gutowski and Reall [25] are written in such a gauge — we will present a detailed analysis of these black holes in the next section 3

A Kaluza-Klein reduction of Page charges to four dimensions for asymptotically flat black holes was given [5]. It is straightforward to do a similar analysis for extremal AdS black holes, but we do not present the details here. Instead, we use the entropy function to compute the four-dimensional physical charges from the near horizon data.

We start with the following ansatz for the near horizon geometry and the horizon gauge field:

$$
\begin{aligned}
d s^{2}= & L_{1}\left(-r^{2} d t^{2}+r^{-2} d r^{2}\right)+L_{2}\left(d \theta^{2}+\sin ^{2} \theta d \psi\right)+L_{3}^{2}[d \phi \\
& \left.\left.+M_{K K} \cos \theta d \psi+z_{1} r d t+A_{K K}(r) d r\right)\right]^{2} \\
A= & q r d t+M_{5} \cos \theta d \psi+P d \phi \\
= & c r d t+M_{4} \cos \theta d \psi+A_{4}(r) d r+P\left[d \phi+z_{1} r d t+M_{K K} \cos \theta+A_{K K}(r) d r\right] .
\end{aligned}
$$

This ansatz is general enough to describe black branes, non-SUSY extremal black holes, and spinning supersymmetric black holes. However, it is not suitable for extremal non-supersymmetric spinning black holes - in this case there is also an angular dependence of some parameters in the near horizon geometry [3, 27].

Due to the presence of the Chern-Simons term, the action is not gauge invariant. To apply the entropy function formalism we have to KK reduce to four dimensions and work with an effective four-dimensional theory - the details of KK reduction of the Chern-Simons term are given in Appendix A.

\footnotetext{
${ }^{3}$ From a physical point of view, it is more natural to use a gauge for which the gauge potential is zero at the horizon. For example, it is well known that the Euclidean time circle shrinks to a point and to avoid any problems one shifts $A_{t}^{H}=0$ by adding a constant. Therefore, $A_{t}^{\infty}=$ constant plays the role of chemical potential of the dual CFT.
} 
The entropy function is (including the $1 / 16 \pi G_{5}$ factor in front of the action)

$$
\begin{aligned}
E= & 2 \pi\left(Q c R+Z z_{1} R-L\right) \\
L= & \frac{R}{16 \pi G_{5}} \int d \theta d \psi \mathcal{L} \\
= & \int_{0}^{\pi} d \theta \int_{0}^{2 \pi} d \psi \frac{R \sin \theta}{18}\left[9 L _ { 1 } L _ { 2 } L _ { 3 } \left(\frac{4\left(c+P z_{1}\right)^{2}}{L_{1}^{2}}-\frac{4\left(P M_{K K}+M_{4}\right)^{2}}{L_{2}^{2}}+\frac{4 L_{2}-L_{3}^{2} M_{K K}^{2}}{L_{2}^{2}}\right.\right. \\
& \left.\left.+\frac{L_{3}^{2} z_{1}^{2}-4 L_{1}}{L_{1}^{2}}+\frac{24}{M^{2}}\right)-16 \sqrt{3} P \zeta\left(P M_{K K}\left(3 c+2 P z_{1}\right)+3 M_{4}\left(2 c+P z_{1}\right)\right)\right] \\
= & \frac{2 \pi R}{9}\left[9 L _ { 1 } L _ { 2 } L _ { 3 } \left(\frac{4\left(c+P z_{1}\right)^{2}}{L_{1}^{2}}-\frac{4\left(P M_{K K}+M_{4}\right)^{2}}{L_{2}^{2}}+\frac{4 L_{2}-L_{3}^{2} M_{K K}^{2}}{L_{2}^{2}}\right.\right. \\
& \left.\left.+\frac{L_{3}^{2} z_{1}^{2}-4 L_{1}}{L_{1}^{2}}+\frac{24}{M^{2}}\right)-16 \sqrt{3} P \zeta\left(P M_{K K}\left(3 c+2 P z_{1}\right)+3 M_{4}\left(2 c+P z_{1}\right)\right)\right] .
\end{aligned}
$$

Here, $R$ is the periodicity of $\phi$ direction, which we would eventually set to identity in the following computations, thus we have $\int d \phi=R=1$.

In five dimensions, there are two conserved charges, namely the electric charge and the angular momentum. In four dimensions, there also are two conserved charges, namely the 4-dimensional electric charge, $Q$, and the electric charge, $Z$, associated to the KK-gauge field. They can be computed in terms of the near horizon parameters, and we obtain

$$
\begin{aligned}
Q & =\frac{3 c L_{2} L_{3}+P\left[3 L_{2} L_{3} z_{1}-2 \sqrt{3} L_{1}\left(P M_{K K}+2 M_{4}\right)\right]}{3 G_{5} L_{1}} \\
Z & =\frac{36 c L_{2} L_{3} P-8 \sqrt{3} L_{1} P^{2}\left(2 P M_{K K}+3 M_{4}\right)+9 L_{2} L_{3} z_{1}\left(L_{3}^{2}+4 P^{2}\right)}{36 G_{5} L_{1}} .
\end{aligned}
$$

These charges are the gauge invariant Maxwell charges that characterize the 4-dimensional solution.

Let us now compute the 5-dimensional Page charges given in (44) and (5). These charges are not gauge invariant, but let us fix the gauge so that the 5 -dimensional gauge field matches (6) :

$$
\begin{aligned}
A_{5} & =A_{4}+P\left(d \phi+A_{k k}\right), \\
F_{a b}^{5} & =F_{a b}^{4}+P F_{a b}^{k k}+\partial_{a} P A_{b}^{k k}-\partial_{b} P A_{a}^{k k} .
\end{aligned}
$$

where $A_{4}$ and $A^{k k}$ can be read off from (6) and $a, b, .$. denote the four-dimensional indices 4 , We obtain the following expression for the electric charge:

$$
\begin{aligned}
Q_{5 d} & =-\frac{1}{4 \pi G_{5}} \int_{H} d \theta d \psi\left[\frac{L_{2} L_{3}}{L 1}\left(c+P z_{1}\right) \sin \theta-\frac{2}{\sqrt{3}} 2 P F_{\psi \theta}^{5}\right] \\
& =-\frac{1}{4 \pi G_{5}} 4 \pi\left[\frac{L_{2} L_{3}}{L 1}\left(c+P z_{1}\right)-\frac{2}{\sqrt{3}} 2\left(P M_{4}+\frac{1}{2} P^{2} M_{k k}\right)\right] \\
& =-\frac{1}{G_{5}}\left[\frac{L_{2} L_{3}}{L 1}\left(c+P z_{1}\right)-\frac{2}{\sqrt{3}}\left(2 P M_{4}+P^{2} M_{k k}\right)\right] .
\end{aligned}
$$

\footnotetext{
${ }^{4}$ For simplicity we consider $\int d \phi=1$ in the ret of this section.
} 
We emphasize that in the Chern-Simons term we have done the integration by parts.

A similar computation can be done for the angular momentum, and we obtain

$$
\begin{aligned}
J_{\xi} & =\frac{1}{4 \pi G_{5}} \int_{H}\left[\frac{1}{4} * \nabla \xi+*(\xi \cdot A) F+\frac{4}{3 \sqrt{3}}(\xi \cdot A) A \wedge F\right] \\
& =-\frac{1}{4 \pi G_{5}} \int_{H} d \theta d \psi\left[-\frac{1}{4}(* \nabla \xi)_{\psi \theta}+P \frac{L_{2} L_{3}}{L_{1}}\left(c+P z_{1}\right) \sin \theta-\frac{4}{3 \sqrt{3}} \frac{3}{2} P^{2} F_{\psi \theta}^{5}\right] \\
& =-\frac{1}{4 G_{5}}\left[\frac{L_{2} L_{3}^{3} z_{1}}{L_{1}}+4 P \frac{L_{2} L_{3}}{L_{1}}\left(c+P z_{1}\right)-4 \frac{2}{3 \sqrt{3}} P^{2}\left(3 M_{4}+2 P M_{k k}\right)\right] .
\end{aligned}
$$

Now, one can compare these results with (8) and so we indeed obtain a match between fourdimensional and five-dimensional charges:

$$
Q_{5 d}=-Q_{4 d}
$$

Thus, we see that the 4-dimensional charges of extremal asymptotically AdS black holes are the dimensional reduced 5-dimensional Page charges for a fixed gauge.

Since in four dimensions there is no Chern-Simons term, the physical charges are the Maxwell charges. These charges are conserved and localized. The $A_{z}$ component of the fivedimensional gauge potential becomes a scalar from a four-dimensional point of view and so the four-dimensional Maxwell charges are shifted because of this term. Since the Page charges are not gauge invariant, they are not the same with the gauge invariant four-dimensional Maxwell charges. However, one can compare them once the gauge is fixed. From a five-dimensional point of view, due to the Chern-Simons term, there is hair (degrees of freedom outside the horizon) which contributes to the charges. In four dimensions, the interpretation is completely different: there is no hair, but there exists an extra scalar which is fixed (due to the attractor mechanism) at the horizon.

\section{$3 \quad$ Examples}

In this section, we apply the previous general results in a few concrete examples. We compute the physical charges and find the near horizon geometry of baryonic and electromagnetic black branes, and also for supersymmetric spinning black holes.

\subsection{Baryonic black branes}

In [23], a new type of black 3-brane charged under a baryonic $U(1)_{B}$ was obtained and a numerical analysis in the limit $T \rightarrow 0$ was presented. However, when the horizon is located deep inside the near $A d S_{2}$ region (small temperature) it is difficult to study the system numerically.

In what follows, we use the results of the previous section to analytically obtain the near horizon data of the baryonic black branes at zero temperature. Our analysis confirms the results of [23]. However, we would like to point out that there is an important problem with this solution. That is, the radius of $A d S_{2}$ is blowing up. 
We are interested in the following effective action, which is a consistent truncation of type IIB SUGRA:

$$
S=\int d^{5} x \sqrt{g}\left[R-\frac{1}{4} e^{2 \eta-\frac{4}{3} \chi} F_{\mu \nu} F^{\mu \nu}-5 \partial_{m u} \eta \partial^{m u} \eta-\frac{10}{3} \partial_{m u} \chi \partial^{m u} \chi-V(\eta, \chi)\right],
$$

where the potential of the two neutral scalars is [23]

$$
V(\eta, \chi)=\frac{8}{L^{2}} e^{-\frac{20}{3} \chi}+\frac{4}{L^{2}} e^{-\frac{8}{3} \chi}\left(e^{-6 \eta}-6 e^{-\eta}\right)
$$

and $L^{4}=4 \pi g_{s} N\left(\alpha^{\prime}\right)^{2} \frac{27}{16}$ is obtained from the quantization of the five-form flux.

We are going to study extremal solutions with the following near horizon geometry:

$$
\begin{array}{r}
d s^{2}=L_{1}\left(-r^{2} d t^{2}+\frac{d r^{2}}{r^{2}}\right)+L_{2}\left(d x^{2}+d y^{2}+d z^{2}\right) \\
A=q r d t, \quad \eta=\eta_{0}, \quad \chi=\chi_{0} .
\end{array}
$$

Here, A is the $U(1)_{B}$ gauge potential; $\eta_{0}$ and $\chi_{0}$ are the horizon values of the neutral scalars. Since we consider a static solution in a theory without Chern-Simons term, there is no fibration in the near horizon geometry. We will see in the next section that, for electromagnetic branes, there is a non-trivial fibration of $A d S_{2}$ in the near horizon geometry.

As usual, the entropy function is $E=2 \pi(q Q-\mathcal{L})$, where $\mathcal{L}$ is the Lagrangian density at the horizon:

$$
\mathcal{L}=\int_{H} d x d y d z \sqrt{g}\left[R-\frac{1}{4} e^{2 \eta-\frac{4}{3} \chi} F_{\mu \nu} F^{\mu \nu}-5 \partial_{m u} \eta \partial^{m u} \eta-\frac{10}{3} \partial_{m u} \chi \partial^{m u} \chi-V(\eta, \chi)\right] .
$$

The equations of motion in the near horizon geometry (attractor equations) are

$$
\begin{aligned}
& L_{2}^{3 / 2} e^{-6 \eta_{0}-\frac{20 \chi_{0}}{3}}\left[\frac{L^{2} q^{2} e^{8 \eta_{0}+\frac{16 \chi_{0}}{3}}}{L_{1}^{2}}-8\left(6 e^{5 \eta_{0}}-1\right) e^{4 \chi_{0}}+16 e^{6 \eta_{0}}\right]=0, \\
& L_{1} \sqrt{L_{2}}\left[\frac{1}{2} e^{-20 \chi_{0} / 3}\left(\frac{q^{2} e^{2 \eta_{0}+\frac{16 \chi_{0}}{3}}}{L_{1}^{2}}-\frac{8\left(\left(1-6 e^{5 \eta_{0}}\right) e^{4 \chi_{0}-6 \eta_{0}}+2\right)}{L^{2}}\right)-\frac{2}{L_{1}}\right]=0 \text {, } \\
& L_{2}^{3 / 2} e^{-6 \eta_{0}-\frac{8 \chi_{0}}{3}}\left[\frac{L^{2} q^{2} e^{8 \eta_{0}+\frac{4 \chi_{0}}{3}}}{L_{1}}-24 L_{1}\left(e^{5 \eta_{0}}-1\right)\right]=0, \\
& L_{2}^{3 / 2} e^{-6 \eta_{0}-\frac{20 \chi_{0}}{3}}\left[\frac{L^{2} q^{2} e^{8 \eta_{0}+\frac{16 \chi_{0}}{3}}}{L_{1}}-16 L_{1}\left(-6 e^{5 \eta_{0}+4 \chi_{0}}+5 e^{6 \eta_{0}}+e^{4 \chi_{0}}\right)\right]=0 \text {, } \\
& Q-\frac{L_{2}^{3 / 2} q e^{2 \eta_{0}-\frac{4 \chi_{0}}{3}}}{L_{1}}=0
\end{aligned}
$$

The last equation relates the gauge potential to the physical (baryonic) charge of the system:

$$
q=\frac{L_{1} Q e^{\frac{4 \chi_{0}}{3}-2 \eta_{0}}}{L_{2}^{3 / 2}}
$$


Next, we rewrite the other equations in terms of $Q$ and solve for the near horizon parameters.

We are interested in regular solutions and so we assume that the radii of $A d S_{2}$ and $R^{3}$ are non-zero quantities (so that we can multiply and divide by $L_{1}, L_{2}$ ). First, we consider combinations of the first two equations and we obtain

$$
\begin{array}{r}
\frac{L^{2}}{L_{1}}+4 e^{-6 \eta_{0}-\frac{20 \chi_{0}}{3}}\left(-6 e^{5 \eta_{0}+4 \chi_{0}}+2 e^{6 \eta_{0}}+e^{4 \chi_{0}}\right)=0 \\
Q^{2} e^{\frac{4 \chi_{0}}{3}-2 \eta_{0}}-\frac{2 L_{2}^{3}}{L_{1}}=0
\end{array}
$$

In this way we get the radii of $A d S_{2}$ and $R^{3}$ in terms of the near horizon values of the scalars:

$$
\begin{aligned}
& L_{1}=-\frac{L^{2} e^{6 \eta_{0}+\frac{20 \chi_{0}}{3}}}{4\left(-6 e^{5 \eta_{0}+4 \chi_{0}}+2 e^{6 \eta_{0}}+e^{4 \chi_{0}}\right)}, \\
& L_{2}=-\frac{L^{2 / 3} Q^{2 / 3} e^{\frac{4}{3}\left(\eta_{0}+2 \chi_{0}\right)}}{2 \sqrt[3]{-6 e^{5 \eta_{0}+4 \chi_{0}}+2 e^{6 \eta_{0}}+e^{4 \chi_{0}}}} .
\end{aligned}
$$

We replace these expressions in the remaining equations of motion and solve for the horizon values of the scalars:

$$
\eta_{0}-4 \chi_{0}=\log \frac{3}{2}, \quad e^{-\eta_{0}}=0 .
$$

We reobtain the results of [23], namely the scalars are blowing up at the horizon for the extremal solutions. This is already a sign that the extremal solution may be problematic.

The advantage of the entropy function is that we can analytically compute the other horizon parameters. First, we observe that even if the horizon values of the scalars diverge, their combination, which enters in the entropy function, is finite. This is why we obtain a finite entropy density

$$
S=\frac{2}{3} \pi Q
$$

which is an indication that the radius of $R^{3}, L_{2}$, is finite - indeed, from the above analysis, we obtain $L_{2}=(L Q / 6)^{2 / 3}$. However, the radius of $A d S_{2}, L_{1}$, is blowing up. This should not come as a surprise, since the horizon values of the moduli are not finite. Therefore we see that $A d S_{2}$ isometry does not exists in the near horizon geometry. Following [27], we would like to comment that for this baryonic black brane system even a flat near-horizon extremal geometry is not possible. It will be interesting to check if in the presence of stringy corrections there exist regular solutions.

\subsection{Stationary black holes}

\subsubsection{Electromagnetic black branes}

A study of black brane solutions of Einstein-Maxwell AdS gravity with a gauge Chern-Simons term can be found in [24, 17]. These solutions are important for understanding how the magnetic fields and Chern-Simons term affect the hydrodynamic properties of the dual field theories. 
In [17, we have used the entropy function to carefully study the near horizon geometry of these black branes at zero temperature and discuss different branches with finite area horizons. We have presented analytic expressions for the entropy density that support the numerical analysis of D'Hoker and Kraus (though, we find a larger class of near horizon geometries). That is, there is a critical value of the magnetic field for which the entropy vanishes.

In this work we are interested to understand the role of the Chern-Simons term for the definition of the physical charges. We know that, due to the Chern-Simons term, the near horizon geometry contains, in fact, a non-trivial fibration of $A d S_{2}$ even if these solutions are not spinning. We start with the near horizon geometry and use the entropy function formalism to compute the four-dimensional charges. Then, we are going to identify the contribution from the Chern-Simons term.

We consider the $z$-direction compactified on a circle of radius $\beta$ and a general $\mathrm{KK}$ ansatz

$$
\begin{aligned}
g_{\alpha \beta} d x^{\alpha} d x^{\beta} & =G_{a b} d x^{a} d x^{b}+G_{A B}\left(d y^{A}+\bar{A}_{a}^{A} d x^{a}\right)\left(d y^{B}+\bar{A}_{a}^{B} d x^{a}\right), \\
A^{(5)} & =A_{\mu}^{(5)} d x^{\mu}=A_{a}^{(4)} d x^{a}+C_{B}\left(x^{a}\right)\left(d y^{B}+\bar{A}_{a}^{B} d x^{a}\right),
\end{aligned}
$$

where $a, b$ are $4 \mathrm{D}$ indices and $A, B$ are compact indices ( $z$ in our case). With this notation we have splitted the coordinates as $x^{\mu}=\left(x^{a}, y^{A}\right)$ and so $A_{\mu}^{(5)}$ is the 5-dimensional gauge potential, $A_{a}^{(4)}$ is the 4-dimensional gauge potential, and $\bar{A}_{a}^{B}$ is the KK gauge potential.

After KK reduction, the metric, gauge field, and relations between the 5-dimensional gauge potential, KK gauge potential, and 4-dimensional gauge potential are

$$
\begin{aligned}
d s^{2} & =L\left(-\frac{d t^{2}}{r^{2}}+r^{2} d r^{2}\right)+v_{1}\left(d x^{2}+d y^{2}\right)+v_{2}\left(d z+z_{1} r d t\right)^{2}, \\
A_{\mu}^{(5)} d x^{\mu} & =\vartheta r d t-\frac{B}{2} y d x+\frac{B}{2} x d y-p_{1}\left(d z+z_{1} r d t\right), \\
F_{r t}^{(5)} & =q=\vartheta-p_{1} z_{1}, \quad F_{x y}^{(5)}=F_{x y}^{(4)}=B, \quad F_{r t}^{(4)}=\vartheta, \quad \bar{F}_{r t}^{z}=z_{1} .
\end{aligned}
$$

The on-shell action and entropy function are

$$
\begin{aligned}
S & =\frac{\mathcal{A}_{x y} \beta}{16 \pi G_{5}}\left[L \sqrt{v_{1}^{2} v_{2}}\left(-\frac{2 B^{2}}{v_{1}^{2}}+\frac{2\left(\vartheta-p_{1} z_{1}\right)^{2}}{L^{2}}+\frac{v_{2} z_{1}^{2}-4 L}{2 L^{2}}+12\right)+8 B p_{1} \zeta\left(\frac{p_{1} z_{1}}{2}-\vartheta\right)\right] \\
\mathcal{E} & =2 \pi \beta \mathcal{A}_{x y}\left(Q \vartheta+\Theta z_{1}-\frac{S}{\beta \mathcal{A}_{x y}}\right)
\end{aligned}
$$

where $Q$ is the 4-dimensional physical charge, $\Theta$ is the physical charge associated to $K K$ gauge field, and $\mathcal{A}_{x y}=\int d x d y$.

The relevant equations are (more details can be found in [17])

$$
\begin{aligned}
\frac{2 \beta \pi}{L}\left[L \Theta+\frac{1}{16 \pi G_{5}}\left(v_{1} \sqrt{v_{2}}\left(4 p_{1} q-v_{2} z_{1}\right)-4 B p_{1}^{2} \zeta L\right)\right] & =0 \\
2 \pi \beta\left[\frac{1}{4 \pi G_{5}}\left(B p_{1} \zeta-\frac{v_{1} \sqrt{v_{2}} q}{L}\right)+Q\right] & =0 .
\end{aligned}
$$


From these equations it is clear that the four-dimensional charges have a contribution that contains the Chern-Simons coupling, $\zeta$. These charges are Maxwell charges and the extra contribution is due to the new scalar associated to the compact direction (the modulus $p_{1}$ ).

To compare with the five-dimensional Page charge, we should compute the expression (4) (at the horizon) by using the near horizon geometry (23). This can be easily done and, as expected, the charges match. As we have already explained, there is a non-trivial fibration of $A d S_{2}$ in the near horizon geometry. The momentum along the $z$-circle becomes, after KK reduction, the 4-dimensional electric charge, $\Theta$. The way the near horizon geometry is fibered will correspond to the magnetic charge. However, since the magnetic charge is topological it can not be fixed by the entropy function.

\subsubsection{Susy spinning AdS black holes}

In flat space [28], the geometry of the event horizon of any supersymmetric black hole of minimal 5-dimensional supergravity must be $T^{3}, S^{1} \times S^{2}$, or a quotient of a homogeneously squashed $S^{3}$. However, there is no general classification of the near horizon geometries of SUSY black holes in AdS spacetime.

In AdS space [25], Gutowski and Reall found an interesting solution that is asymptotically AdS and does not have an $A d S_{3}$ component in the near-horizon geometry. In the ungauged theory the near-horizon geometry of a BPS black hole is maximally supersymmetric. In the gauged supergravity this is not true because the only maximally supersymmetric solution is $A d S_{5}$.

We use the coordinates that make the $A d S_{2}$ part of the near horizon geometry manifest [19]:

$$
\begin{aligned}
d s^{2} & =\frac{1}{\Delta^{2}+9 \ell^{-2}}\left(-r^{2} d \tau^{2}+r^{-2} d r^{2}\right)+\frac{1}{\Delta^{2}-3 \ell^{-2}}\left(d \theta^{2}+\sin ^{2} \theta d \psi^{2}\right) \\
& +\left(\frac{\Delta}{\Delta^{2}-3 \ell^{-2}}\right)^{2}\left[d \phi+\cos \theta d \psi-\frac{3 r \alpha}{\ell \Delta} \frac{\Delta^{2}-3 \ell^{-2}}{\Delta^{2}+9 \ell^{-2}}\left(d \tau+\frac{d r}{r^{2}}\right)\right]^{2}
\end{aligned}
$$

and the gauge potential

$$
A=\frac{\sqrt{3}}{2\left(\Delta^{2}+9 l^{-2}\right)} \Delta r d \tau-\frac{\sqrt{3} \alpha \cos \theta}{2 \ell\left(\Delta^{2}-3 \ell^{-2}\right)} d \psi
$$

where, $\Delta>\frac{\sqrt{3}}{\ell}$ and $\alpha= \pm 1$.

Since we have an exact solution, our goal is not to use the entropy function for finding the near horizon geometry. We want to use the entropy function to compute the corresponding 4dimensional (physical) Maxwell charges from the attractor equations. First, we do KK reduction to obtain a gauge invariant effective action. Then, we check that the near horizon geometry of Gutowski-Reall black hole is a solution. Finally, we solve the gauge fields' equations of motion to obtain the physical charges from the near horizon geometry.

It is straightforward to check that the near horizon geometry and gauge field (26, 32) are solutions of the attractor equations obtained from the general ansatz (6) $)$. 
Before presenting the charges, let us make a couple of observations. First, by using the equations of motion for $L_{1}$ and $L_{2}$ we obtain that $L_{1}\left(12 L_{2}+l^{2}\right)-L_{2} l^{2}=0$. This is expected [13, since in $A d S$ spacetime the radii of $A d S_{2}$ and $S^{3}$ in the near horizon geometry are not equal - they are related by the cosmological constant (more generally, by the potential of the moduli). The second observation is related to the entropy. That is, the parameter $P$ can not be fixed by the equations of motion in the near horizon limit. The reason is that in the work [25] the convention for the Chern-Simons coefficient is $\zeta=1$ and the equation of $P$ is trivially satisfied in this way. One may think that the fact that $P$ is not fixed causes problems for the entropy $-P$ appears in the entropy function and is not fixed. However, a concrete computation of the entropy reveals that $P$ cancels trivially and does not appear in the final expression of the entropy:

$$
S=\frac{16 \pi^{2} R l^{4} \Delta}{\left(l^{2} \Delta^{2}-3\right)^{2}}
$$

where $R$ is the periodicity of $\phi$.

However, the expressions of the 4-dimensional charges depend on $P$. Since we have an exact solution we read off $P$ from the near horizon geometry and get:

$$
P=\frac{\sqrt{3} M \alpha}{6-2 M^{2} \Delta^{2}}
$$

We also obtain the following 4-dimensional charges:

$$
Q=\frac{\sqrt{3} M^{2}\left(M^{2} \Delta^{2}-1\right)}{2 G_{5}\left(M^{2} \Delta^{2}-3\right)^{2}}, \quad Z=\frac{M^{3} \alpha\left(1-3 M^{2} \Delta^{2}\right)}{2 G_{5}\left(M^{2} \Delta^{2}-3\right)^{3}} .
$$

These charges should be used when comparing the thermodynamical and statistical entropies.

For completeness, let us also present the expressions of the five dimensional charges: 5

$$
Q_{5 d}=\frac{\sqrt{3} r_{0}^{2}\left(2 M^{2}+r_{0}^{2}\right)}{16 G_{5} M^{2}}, \quad J=\frac{r_{0}^{4} \alpha\left(3 M^{2}+2 r_{0}^{2}\right)}{32 G_{5} M^{3}}
$$

where $r_{0}$ is the horizon radius. We notice that while $Q_{5 d}$ is independent of the parameter $\alpha(= \pm 1)$, the angular momentum $J$ does depend on it. Now, using the relation between the horizon radius $r_{0}$ and near horizon parameter $\Delta, r_{0}=2 M \sqrt{\frac{1}{M^{2} \Delta^{2}-3}}$, we get the expected match between the four-dimensional and five-dimensional charges. We would like to point out that the solution is written in a gauge for which the Page charge matches the electric five dimensional Maxwell charge.

\section{Discussion}

We have used the entropy function formalism to compute the physical charges of a general class of extremal AdS black holes. These new results combined with the method of [5] prove

\footnotetext{
${ }^{5}$ Compared to [25, our definitions for the five-dimensional charges should be divided by $4 \pi$. The reason is that we have considered the periodicity of $\phi, R=1$, in the previous general discussion in Section 2 .
} 
that, indeed, the physical charges in the presence of the gauge Chern-Simons term are the Page charges. This closes a gap in computing the physical charges from the near horizon data for extremal AdS black holes.

The focus of this paper was on the gauge Chern-Simons term and we did not consider the gravitational Chern-Simons term, which is a four derivative metric term. One expects that again the charges will be shifted in a similar manner. Indeed, the shift in four-dimensional and five-dimensional charges found in [10, 21] is proportional to the above mentioned term. Their analysis was done for asymptotically flat black holes.

We then checked our results in some explicit examples. In particular, we have obtained analytic results for the near horizon geometry of baryonic branes at zero temperature. Since these are extremal solutions, the near horizon geometry contains an $A d S_{2}$ spacetime. We have found that the radius of $A d S_{2}$ is blowing up, and from this point of view the solution is problematic. However, it still remains possible that a general set of stringy corrections would allow regular solutions in this case.

An analysis of the physics of AdS black holes using the near horizon data only is also important for computing the shear viscosity to entropy density ratio due to the AdS/CFT duality (for other transport coefficients there are, in general, non-trivial flows and so the near horizon geometry is not sufficient). In the presence of the gauge Chern-Simons term, one has to work with the right physical charges in five dimensions. These charges are the Page charges (not the usual Maxwell charges) and we have proven that they consistently match the physical charges obtained from the entropy function. Due to the Chern-Simons term, there is a non-trivial fibration of $A d S_{2}$ in the near horizon geometry.

When there is a compact direction, after KK reduction, we can compare the five dimensional Page charges with the Maxwell charges in four dimensions. We have found that, indeed, they match as suggested by [5]. However, our interpretation is slightly different. The reason is that the entropy function formalism provides enough information to exactly understand the meaning of the extra contribution from the gauge Chern-Simons term. Since we have presented in detail these arguments in Section 2, here we would like just to point out that the interpretation is completely different in four dimensions. That is, there is no hair, but there exists an extra scalar that is fixed (due to the attractor mechanism) at the horizon. The extra contributions are due to this modulus.

Finally, we have also investigated SUSY spinning black holes in AdS. We have computed the charges for an exact solution and found again the expected agreement. This solution has a spherical horizon and one can wonder how our results will apply to spinning black holes with toroidal horizon geometry. If one can find such a solution, it will be also interesting to compute the transport coefficients of the dual plasma. Unfortunately, this solution does not exist 6 This observation was made in [25], where it was proven that the corresponding supersymmetric nearhorizon solution joins, in fact, to a solution which is asymptotically a plane-wave geometry and not AdS as we need. In Appendix B, we use the method of [25] to show that there can not exist an asymptotically AdS solution, which can join up with this near horizon geometry and preserves $\frac{1}{4}$ SUSY (which is minimal in this case). Though, as a final remark, we would like

\footnotetext{
${ }^{6}$ We would like to thank Harvey Reall for clarifications on this point.
} 
to point out that there may exist non-supersymmetric extremal spinning AdS black holes with toroidal horizon geometry.

\section{Acknowledgements}

We would like to thank Guillaume Bossard, Yuji Tachikawa, Stefan Theisen, Stefan Vandoren, Oscar Varela and Bernard de Wit for interesting discussions and Ashoke Sen for valuable discussions and comments on an earlier draft of this paper. DA would also like to thank PUCV Chile, Universidad Catolica de Chile, and CECS Valdivia for the hospitality during part of this work and the audience at all these places for their positive feedback. Work of NB is supported by NWO Veni grant, the Netherlands.

\section{Appendix}

\section{A KK reduction of Chern-Simons term}

We would like to use entropy function formalism to compute the physical charges. Since the 5-dimensional action is not gauge invariant, we should KK reduce to four dimensions. In this section we explicitly show the KK reduction of the Chern-Simons term.

First, we observe that the most general gauge potential compatible with the symmetries of the metric (26) is

$$
A=\frac{\sqrt{3}}{2\left(\Delta^{2}+9 l^{-2}\right)} \Delta r d \tau-\frac{\sqrt{3} \cos \theta}{2 \ell\left(\Delta^{2}-3 \ell^{-2}\right)} d \psi+b\left[d \phi+\cos \theta d \psi-\frac{3 r}{\ell \Delta} \frac{\Delta^{2}-3 \ell^{-2}}{\Delta^{2}+9 \ell^{-2}}\left(d \tau+\frac{d r}{r^{2}}\right)\right] .
$$

Unlike the other cases (e.g., SUSY black holes in flat space) studied in the literature, the KK gauge potential has also a magnetic part. Since $A_{r}^{K K}$ is a function of the radial coordinate only, it does not play any role in our analysis (contributions of the form $\epsilon^{\mu \nu \alpha r r} \partial_{r} A_{r}$ are zero in the Chern-Simons term).

Let us consider a generic ansatz for the gauge potential:

$$
A=A_{\mu} d x^{\mu}=B_{a} d x^{a}+b\left(x^{a}\right)\left[d \phi+A_{a}^{K K} d x^{a}\right]
$$

where by $\mu, \nu, .$. we denote the 5 -dimensional indices and by $a, b, .$. the 4 -dimensional ones. The Chern-Simons term becomes

$$
\epsilon^{\alpha \beta \gamma \delta \mu} A_{\alpha} F_{\beta \gamma} F_{\delta \mu}=3 \epsilon^{\beta \gamma \delta \mu} A_{\phi} F_{\beta \gamma} F_{\delta \mu}
$$

and its contribution to the action is

$$
\begin{aligned}
C S & =\frac{\zeta}{16 \pi G_{5}} R \int d^{4} x \epsilon^{a b c d} A_{\phi} F_{a b} F_{c d}, \\
F_{a b} & =\partial_{a} A_{b}-\partial_{b} A_{a} .
\end{aligned}
$$


where $R$ is the periodicity of $\phi$ (KK compact direction).

To rewrite this part in a suitable form, one should also integrate by parts terms of the form $b \partial_{\mu} b$ or $b^{2} \partial_{\mu} b$. The final result (in a covariant frame) is

$$
C S=\frac{4 R \zeta}{16 \pi G_{5}} \int d^{4} x \epsilon^{a b c d}\left[\frac{1}{3} b^{3} \partial_{a} A_{b}^{K K} \partial_{c} A_{d}^{K K}+b \partial_{a} B_{b} \partial_{c} B_{d}+b^{2} \partial_{a} A_{b}^{K K} \partial_{c} B_{d}\right] .
$$

To illustrate this method, let us consider a simple example when there is no KK magnetic charge:

$$
A=e r d \tau+p \cos \theta d \psi+b\left[\bar{e} r d \tau+A_{r}(r) d r\right] .
$$

The relevant part of the Chern-Simons term is

$$
b F_{r t} F_{\theta \psi}=b p \partial_{r} A_{\tau}=p\left[b e+\bar{e} b \partial_{r}(b r)\right]=p\left[b e+\bar{e}\left(b^{2}+b r \partial_{r} b\right)\right]=p\left[b e+\bar{e}\left(b^{2}+\frac{r}{2} \partial_{r} b^{2}\right)\right] .
$$

The last term is then $b^{2}-b^{2} / 2+\partial_{r}\left(r b^{2}\right) / 2$ and we see that after we get rid of the total derivative, we obtain $p b(e+b \bar{e} / 2)$.

\section{B Toroidal Spinning Black Brane}

In this appendix, we will discuss another possible near-horizon geometry that arises in five-

dimensional minimal gauged supergravity, namely $A d S_{2} \times T^{2} \times S^{1}$. This corresponds to $\Delta=\frac{\sqrt{3}}{\ell}$ [25]:

$$
\begin{aligned}
d s^{2} & =-\frac{3 r^{2}}{\ell^{2}} d u^{2}+2 d u d r-\frac{6 r}{\ell} d u\left(d z+\frac{\sqrt{3}}{2 \ell}(y d x-x d y)\right) \\
& +\left(d z+\frac{\sqrt{3}}{2 \ell}(y d x-x d y)\right)^{2}+d x^{2}+d y^{2}, \\
F & =-\frac{3}{2 \ell} d u \wedge d r+\frac{\sqrt{3}}{2 \ell} d x \wedge d y .
\end{aligned}
$$

This metric can be rewritten as

$$
d s^{2}=-\frac{12 r^{2}}{l^{2}} d u^{2}+2 d u d r+\left(\left(d z+\frac{\sqrt{3}}{2 \ell}(y d x-x d y)\right)-\frac{3 r}{l} d u\right)^{2}+d x^{2}+d y^{2}
$$

and with the following coordinate transformation

$$
\tau=\frac{12}{l^{2}} u+\frac{1}{r}
$$

we explicitly obtain the $A d S_{2}$ part:

$$
d s^{2}=\frac{l^{2}}{12}\left(-r^{2} d \tau^{2}+\frac{d r^{2}}{r^{2}}\right)+\left(\left(d z+\frac{\sqrt{3}}{2 \ell}(y d x-x d y)\right)-\frac{l r}{4}\left(d \tau+\frac{d r}{r^{2}}\right)\right)^{2}+d x^{2}+d y^{2}
$$


The gauge field strength becomes

$$
F=-\frac{3 l}{24} d \tau \wedge d r+\frac{\sqrt{3}}{2 \ell} d x \wedge d y
$$

In [25], the authors have shown that this supersymmetric near horizon geometry corresponds to a solution, which is a plane-wave geometry. In this appendix we will show that there is no asymptotically AdS with this near horizon geometry such that it preserves $\frac{1}{4}$ SUSY. To show this, we will proceed as in [25]. We will try to construct a base space solution that can give us a global AdS black brane geometry. It was proved in [26] that a SUSY solution of this theory can be completely parameterized in terms of a scalar $f$, a real vector $V^{\alpha}$, and three real two-form fields $X^{i}$. These quantities satisfy the following algebraic relations:

$$
\begin{aligned}
V^{\alpha} V_{\alpha} & =-f^{2}, \\
X^{i} \wedge X^{j} & =-2 \delta_{i j} f \star V, \\
\iota_{V} X^{i} & =0, \\
\iota_{V} \star X^{i} & ==-f X^{i}, \\
\left(X^{i}\right)_{\gamma \alpha}\left(X^{J}\right)_{\beta}^{\gamma} & =\delta_{i j}\left(f^{2} \eta_{\alpha \beta}+V_{\alpha} V_{\beta}\right)-\epsilon_{i j k} f\left(X^{k}\right)_{\alpha \beta} .
\end{aligned}
$$

They also satisfy the following differential relations:

$$
\begin{aligned}
d f & =-\frac{2}{\sqrt{3}} \iota_{V} F \\
\left.D_{(\alpha} V_{\beta}\right) & =0 \\
d V & =-\frac{4}{\sqrt{3}} f F-\frac{2}{\sqrt{3}} \star(F \wedge V)-2 l^{-1} X^{1}, \\
d X^{i} & =\frac{1}{l} \epsilon_{1 i j}\left[2 \sqrt{3} A \wedge X^{j}+3 \star X^{j}\right] .
\end{aligned}
$$

We would like to construct a solution for which $V$ is globally defined and it can always be made timelike in a coordinate patch. In such a coordinate patch, the metric can be written in a nice form

$$
d s_{5}^{2}=-f^{2}(d t+\omega)^{2}+f^{-1} d s_{4}^{2},
$$

and without the loss of generality the scalar $f$ can be chosen to be positive and $V=\frac{\partial}{\partial t}$. Here, $d s_{4}^{2}$ is the metric of the base space $\mathcal{B}$ and $\omega$ is a one-form on $\mathcal{B}$. Imposing the supersymmetric constraints listed above and integrability, one finds that $\mathcal{B}$ has to be a Kahler manifold with $X^{1}$ the anti-selfdual Kahler form. Also the scalar $f=-\frac{24}{R}$, where $R$ is the Ricci scalar of the base space. Now let us first write down the Kahler base space metric and the corresponding Kahler for the near horizon geometry (39)

$$
\begin{aligned}
d s_{4}^{2} & =d r^{2}+r^{2}\left(\sigma_{1}^{2}+\sigma_{2}^{2}\right)+4 r^{2} \sigma_{3}^{2} \\
X^{1} & =\sqrt{3} d\left[r^{2} \sigma_{3}\right],
\end{aligned}
$$

where we have defined three one-forms

$$
\sigma_{1}=\sqrt{3} d x, \quad \sigma_{2}=\sqrt{3} d x, \quad \sigma_{3}=\sqrt{3}\left(d z+\frac{\sqrt{3}}{2}(y d x-x d y)\right) .
$$


Next, we write the base space and the two-form corresponding to the asymptotically AdS space that joins up with the near horizon metric (39) as

$$
\begin{aligned}
d s_{4}^{2} & =d r^{2}+A^{2}(r)\left(\sigma_{1}^{2}+\sigma_{2}^{2}\right)+4 B(r)^{2} \sigma_{3}^{2} \\
X^{1} & =d\left[C(r) \sigma_{3}\right]
\end{aligned}
$$

where $A(r), B(r), C(r)$ are arbitrary functions of the radial coordinate $\mathrm{r}$ so that, near the horizon, they are given by (47).

Since the AdS spacetime is maximally symmetric, it requires the gauge field strength to vanish and due to first equation of (45) the scalar $f$ is constant. Without loss of generality, we can choose it to be identity. Now, the anti-selfduality of $X^{1}$ gives

$$
C(r)=A(r)^{2}, \quad B(r)=2 A^{\prime}(r) A(r) .
$$

Thus effectively we have to find only one function $A(r)$ to fix the geometry. Moreover, since the scalar function $f$ is a constant in this case and has been set to one, the Ricci scalar $R$ of the base space metric should be a constant, namely $R=-24$. Since the Ricci scalar is

$$
R=-\frac{2\left(4 A^{\prime}(r)^{2}+7 A(r) A^{\prime \prime}(r)\right)}{A(r)}-\frac{2 A(r)^{3}}{A^{\prime}(r)}
$$

a solution is $A(r)=e^{r}$.

It was shown in 25] that another necessary condition for the geometry to be asymptotically AdS is

$$
R_{i j p q}=-2 X_{i j}^{1} X_{p q}^{1}+\left(X_{q i}^{1} X_{p j}^{1}-X_{p i}^{1} X_{q j}^{1}\right)-\left(\delta_{p i} \delta_{q j}-\delta_{q i} \delta_{p j}\right)
$$

where $(i, j, p, q)$ are tangent space indices. This condition gets satisfied automatically with the above choice of the metric function $A(r)=e^{r}$. The SUSY geometry can then be written as

$$
d s^{2}=-\left(d t+2 e^{2 r} \sigma_{3}\right)^{2}+3 e^{2 r}\left(d x^{2}+d y^{2}+4 e^{2 r}\left(d z+\frac{\sqrt{3}}{2}(y d s-x d y)\right)^{2}\right)+d r^{2}
$$

With a coordinate transformation $e^{r}=\rho$ we can easily see that it is a PP-wave geometry:

$$
\begin{aligned}
d s^{2}= & -\left(d t+2 \rho^{2} \sqrt{3}\left(d z+\frac{\sqrt{3}}{2}(y d s-x d y)\right)\right)^{2}+\frac{d \rho^{2}}{\rho^{2}} \\
& +3 \rho^{2}\left(d x^{2}+d y^{2}\right)+12 \rho^{4}\left(d z+\frac{\sqrt{3}}{2}(y d s-x d y)\right)
\end{aligned}
$$

Therefore, we conclude that there is no asymptotically AdS supersymmetric spinning black brane solution with (39) as the near-horizon geometry. 


\section{References}

[1] A. Sen, "Black Hole Entropy Function and the Attractor Mechanism in Higher Derivative Gravity," JHEP 0509, 038 (2005) arXiv:hep-th/0506177].

[2] A. Sen, "Entropy function for heterotic black holes," JHEP 0603, 008 (2006) arXiv:hep-th/0508042.

[3] D. Astefanesei, K. Goldstein, R. P. Jena, A. Sen and S. P. Trivedi, "Rotating attractors," JHEP 0610, 058 (2006) arXiv:hep-th/0606244.

[4] A. Sen, "Black Hole Entropy Function, Attractors and Precision Counting of Microstates," Gen. Rel. Grav. 40, 2249 (2008) [arXiv:0708.1270 [hep-th]].

[5] K. Hanaki, K. Ohashi and Y. Tachikawa, "Comments on charges and near-horizon data of black rings," JHEP 0712, 057 (2007) [arXiv:0704.1819 [hep-th]].

[6] K. Copsey and G. T. Horowitz, "The role of dipole charges in black hole thermodynamics," Phys. Rev. D 73, 024015 (2006) [arXiv:hep-th/0505278];

D. Astefanesei and E. Radu, "Quasilocal formalism and black ring thermodynamics," Phys. Rev. D 73, 044014 (2006) arXiv:hep-th/0509144;

R. Emparan, "Rotating circular strings, and infinite non-uniqueness of black rings," JHEP 0403, 064 (2004) arXiv:hep-th/0402149.

[7] D. Astefanesei, R. B. Mann, M. J. Rodriguez and C. Stelea, "Quasilocal formalism and thermodynamics of asymptotically flat black objects," Class. Quant. Grav. 27, 165004 (2010) arXiv:0909.3852 [hep-th]];

D. Astefanesei, M. J. Rodriguez and S. Theisen, "Quasilocal equilibrium condition for black ring," JHEP 0912, 040 (2009) arXiv:0909.0008 [hep-th]].

[8] J. C. Breckenridge, R. C. Myers, A. W. Peet, C. Vafa, "D-branes and spinning black holes," Phys. Lett. B391, 93-98 (1997). |hep-th/9602065|.

[9] N. Banerjee, "Subleading Correction to Statistical Entropy for BMPV Black Hole," Phys. Rev. D 79, 081501 (2009) arXiv:0807.1314 [hep-th]].

[10] A. Castro and S. Murthy, "Corrections to the statistical entropy of five dimensional black holes," JHEP 0906, 024 (2009) arXiv:0807.0237 [hep-th]].

[11] N. Banerjee, I. Mandal and A. Sen, "Black Hole Hair Removal," JHEP 0907, 091 (2009) arXiv:0901.0359 [hep-th]].

D. P. Jatkar, A. Sen and Y. K. Srivastava, "Black Hole Hair Removal: Non-linear Analysis," JHEP 1002, 038 (2010) [arXiv:0907.0593 [hep-th]].

[12] D. Marolf, "Chern-Simons terms and the three notions of charge," arXiv:hep-th/0006117. 
[13] D. Astefanesei, H. Nastase, H. Yavartanoo and S. Yun, "Moduli flow and nonsupersymmetric AdS attractors," JHEP 0804, 074 (2008) [arXiv:0711.0036 [hep-th]].

[14] N. Iqbal and H. Liu, "Universality of the hydrodynamic limit in AdS/CFT and the membrane paradigm," Phys. Rev. D 79, 025023 (2009) arXiv:0809.3808 [hep-th]].

[15] N. Banerjee and S. Dutta, "Higher Derivative Corrections to Shear Viscosity from Graviton's Effective Coupling," JHEP 0903, 116 (2009) arXiv:0901.3848 [hep-th]].

[16] N. Banerjee and S. Dutta, "Near-Horizon Analysis of eta/s," Nucl. Phys. B 845, 165 (2011) arXiv:0911.0557 [hep-th]].

[17] D. Astefanesei, N. Banerjee and S. Dutta, "Moduli and electromagnetic black brane holography," arXiv:1008.3852 [hep-th].

[18] N. Banerjee and S. Dutta, JHEP 1008, 041 (2010) [arXiv:1005.2367 [hep-th]].

[19] D. Astefanesei, Y. K. Srivastava, "CFT Duals for Attractor Horizons," Nucl. Phys. B822, 283-300 (2009). arXiv:0902.4033 [hep-th]].

[20] G. L. Cardoso, J. M. Oberreuter and J. Perz, "Entropy function for rotating extremal black holes in very special geometry," JHEP 0705, 025 (2007) arXiv:hep-th/0701176.

[21] B. de Wit and S. Katmadas, "Near-horizon analysis of D=5 BPS black holes and rings," JHEP 1002, 056 (2010) [arXiv:0910.4907 [hep-th]].

[22] J. F. Morales and H. Samtleben, "Entropy function and attractors for AdS black holes," JHEP 0610, 074 (2006) [arXiv:hep-th/0608044];

D. Astefanesei, N. Banerjee, S. Dutta, "(Un)attractor black holes in higher derivative AdS gravity," JHEP 0811, 070 (2008). arXiv:0806.1334 [hep-th]];

F. W. Shu and X. H. Ge, "Entropy function and higher derivative corrections to entropies in (anti-)de Sitter space," JHEP 0808, 021 (2008) arXiv:0804.2724 [hep-th]];

J. Choi, S. Lee and S. Lee, "Near Horizon Analysis of Extremal AdS5 Black Holes," JHEP 0805, 002 (2008) [arXiv:0802.3330 [hep-th]].

[23] C. P. Herzog, I. R. Klebanov, S. S. Pufu and T. Tesileanu, "Emergent Quantum NearCriticality from Baryonic Black Branes," JHEP 1003, 093 (2010) arXiv:0911.0400 [hepth]].

[24] E. D'Hoker, P. Kraus, "Magnetic Brane Solutions in AdS," JHEP 0910, 088 (2009). arXiv:0908.3875 [hep-th]];

E. D'Hoker, P. Kraus, "Charged Magnetic Brane Solutions in AdS (5) and the fate of the third law of thermodynamics," JHEP 1003, 095 (2010). arXiv:0911.4518 [hep-th]];

E. D'Hoker, P. Kraus, "Holographic Metamagnetism, Quantum Criticality, and Crossover Behavior," JHEP 1005, 083 (2010). arXiv:1003.1302 [hep-th]];

E. D'Hoker, P. Kraus, A. Shah, "RG Flow of Magnetic Brane Correlators," arXiv:1012.5072 [hep-th]]. 
[25] J. B. Gutowski, H. S. Reall, "Supersymmetric AdS(5) black holes," JHEP 0402, 006 (2004). [hep-th/0401042].

[26] J. P. Gauntlett, J. B. Gutowski, C. M. Hull, S. Pakis and H. S. Reall, Class. Quant. Grav. 20, 4587 (2003) arXiv:hep-th/0209114.

[27] H. K. Kunduri, J. Lucietti and H. S. Reall, "Near-horizon symmetries of extremal black holes," Class. Quant. Grav. 24, 4169 (2007) [arXiv:0705.4214 [hep-th]];

D. Astefanesei and H. Yavartanoo, "Stationary black holes and attractor mechanism," Nucl. Phys. B 794, 13 (2008) [arXiv:0706.1847 [hep-th]].

[28] H. S. Reall, "Higher dimensional black holes and supersymmetry," Phys. Rev. D68, 024024 (2003). [hep-th/0211290]. 